\title{
Multiple carcinogenesis contributes to the heterogeneity of HCC
}

\author{
Qiang Gao, Xiao-Ying Wang, Jian Zhou and Jia Fan
}

Shibata and Aburatani ${ }^{1}$ (Exploration of liver cancer genomes. Nat. Rev. Gastroenterol. Hepatol. 11, 340-349 [2014]) reviewed the latest understanding of the genomic profile of hepatocellular carcinoma (HCC) using next-generation sequencing approaches. We agree with the authors that the molecular pathogenesis of HCC is extremely complex and heterogeneous, and molecular information should guide HCC drug development. Considering that multiple carcinogenesis is a major characteristic of HCC, we would like to raise some further points for discussion.

Multifocal HCC can arise synchronously or metachronously as new tumours (multicentric occurrence) or as intrahepatic metastases of the primary cancer. ${ }^{2,3}$ In two patients with HBV infection and multifocal HCC, Miao et al. ${ }^{4}$ found that the primary cancer, portal vein tumour thrombus (PVTT) and all three intrahepatic metastases had similar mutation patterns in all regions (coding, noncoding, genic and intergenic), whereas ratios of nonsynonymous to synonymous substitution were significantly higher in the metastatic lesions (1.49) than in the primary tumour $(0.96)(P<0.05)$. This finding indicates a positive Darwinian selection favouring the growth of metastatic subclones. By contrast, two tumours from a patient with multicentric occurrence had distinct mutation profiles, but with comparable nonsynonymous to synonymous substitution rates ( 0.83 versus 0.81$).{ }^{4}$ Similarly, in HCV-related multifocal HCC, ${ }^{5}$ whole-exome sequencing revealed no commonly mutated genes in two multicentric tumours from one patient, with 29 and 225 independent somatic mutations in each tumour, respectively. However, in two other patients, ${ }^{5} 32$ (64.0\%) and nine (24.3\%) mutated genes were shared in their synchronous tumours, respectively, suggesting an origin from common tumour-precursor cells or through intrahepatic dissemination.

We identified 5, 72 and 180 somatic mutations in a dysplastic nodule and two tumours, respectively, in a patient with HBV infection and HCC. ${ }^{6}$ MUC16 and
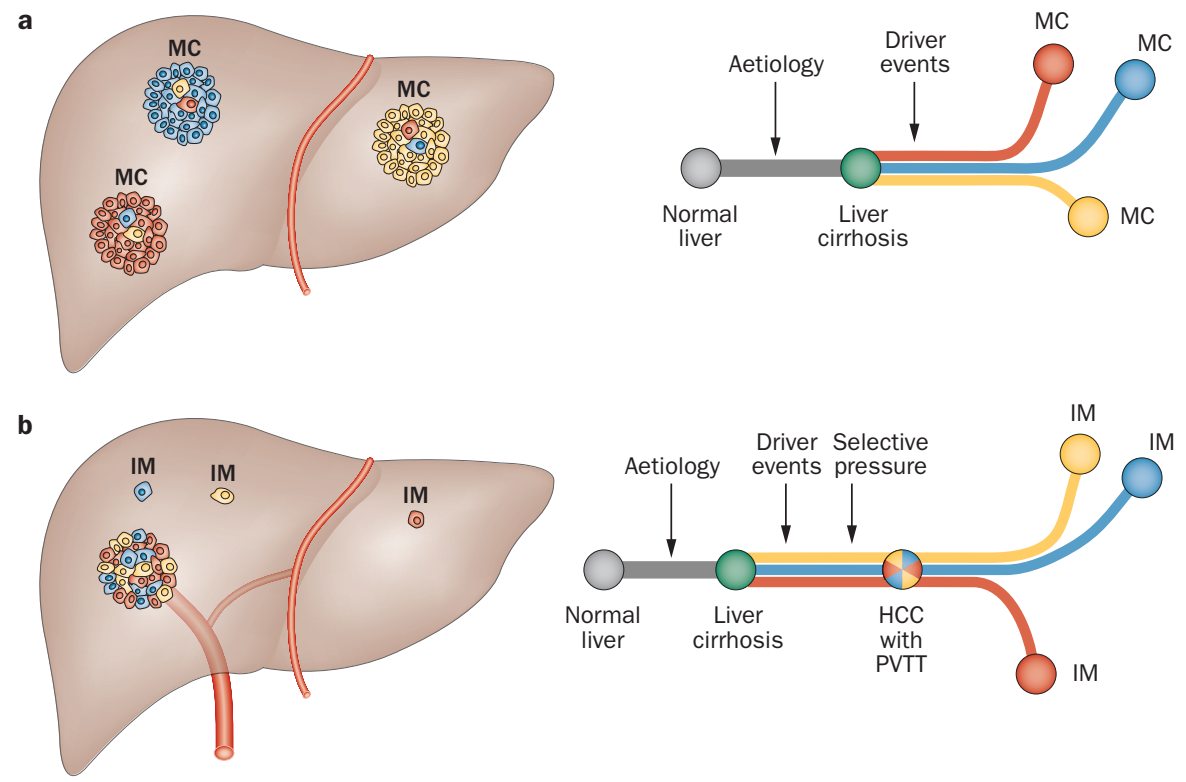

Figure 1 | Simplified evolution of multicentric occurrence and intrahepatic metastatic HCC. a Multicentric HCC develops through accumulation of highly different sets of genetic alterations (although these alterations share the same genetic and environmental backgrounds). b | The primary HCC spreads along the portal vein to form a tumour thrombus, resulting in extensive intrahepatic metastases. Circles in different colours represent genetically distinct clones. Branch colours indicate different sets of genetic alterations. Abbreviations: IM, intrahepatic metastasis; MC, multicentric occurrence.

UBE3C were commonly mutated, but in distinct exonic regions and in both tumours rather than in the dysplastic nodule, indicating the nature of multicentric occurrence. Similarly, whole-genome sequencing of two pairs of multicentric tumours in patients with HCV-related HCC showed no common somatic mutations or structural alterations. ${ }^{7}$ ATM, FSIP2 and LRFN5 were mutated in both tumours of one patient, but in different locations of the genes. Notably, the pairs of each multicentric tumour clustered together with respect to overall mutational spectra, indicating similar somatic substitution patterns at the whole-genome level.

PVTT marks the typical onset of HCC with intrahepatic metastasis. ${ }^{8}$ Whole-exome sequencing of nine pairs of matched primary tumour and PVTT revealed that 324 (94.2\%) mutations were identical in these paired tumours and PVTTs, whereas four (1.1\%) and $16(4.7 \%)$ were detected only in primary tumours or PVTTs, respectively, suggesting that PVTTs inherit most genetic alterations from primary tumours. ${ }^{9}$ Whole-genome sequencing of a primary HCC and two metachronously developed tumours after resection ${ }^{10}$ showed that 205 (95.8\%) mutations were observed at similar frequencies across all three tumours, with nine $(4.2 \%)$ observed at varying frequencies among tumours, indicating that these tumours were highly clonal. Interestingly, four different cell lineages were detected in the primary HCC, and the two recurrent tumours derived from one of the lineages each.

Collectively, these findings indicate that multicentric tumours develop through an accumulation of highly different sets of genetic alterations, whereas intrahepatic metastases share similar genetic profiles (Figure 1) to the primary tumour. Surprisingly, 


\section{CORRESPONDENCE}

$30-70 \%$ of synchronous or metachronous tumours are independent lesions, ${ }^{2,3,11}$ which is important when considering treatment selection. For example, patients with intrahepatic metastases could receive targeted therapy based on the molecular profile of the original tumour, whereas patients with multicentric tumours might benefit from managing the underlying liver disease, considering that these tumours share the same genetic and environmental backgrounds. Assessing the pathogenesis of all tumours by multi-omics profiling might provide a full view of the heterogeneous genomic landscape of HCC to facilitate personalized management.

Liver Cancer Institute, Zhongshan Hospital and Shanghai Medical School, Fudan University, 180 Fenglin Road, Shanghai 200032, P. R. China (G.Q., X.-Y.W., J.Z., J.F.) Correspondence to: J.F.

fan.jia@zs-hospital.sh.cn
Acknowledgements

We would like to thank Dr Y.-Z. He for his expertise in drafting Figure 1.

\section{Competing interests}

The authors declare no competing interests.

1. Shibata, T. \& Aburatani, H. Exploration of liver cancer genomes. Nat. Rev. Gastroenterol. Hepatol. 11, 340-349 (2014).

2. Ng, I. O., Guan, X. Y., Poon, R. T., Fan, S. T. \& Lee, J. M. Determination of the molecular relationship between multiple tumour nodules in hepatocellular carcinoma differentiates multicentric origin from intrahepatic metastasis. J. Pathol. 199, 345-353 (2003).

3. Oikawa, T. et al. Multistep and multicentric development of hepatocellular carcinoma: histological analysis of 980 resected nodules. J. Hepatol. 42, 225-229 (2005).

4. Miao, R. et al. Identification of prognostic biomarkers in hepatitis B virus-related hepatocellular carcinoma and stratification by integrative multi-omics analysis. J. Hepatol. 61 840-849 (2014).

5. Ikeda, A. et al. Leptin receptor somatic mutations are frequent in HCV-infected cirrhotic liver and associated with hepatocellular carcinoma. Gastroenterology 146, 222-232 e235 (2014).

6. Jiang, J. H. et al. Clinical significance of the ubiquitin ligase UBE3C in hepatocellular carcinoma revealed by exome sequencing. Hepatology 59, 2216-2227 (2014).

7. Fujimoto, A. et al. Whole-genome sequencing of liver cancers identifies etiological influences on mutation patterns and recurrent mutations in chromatin regulators. Nat. Genet. 44, 760-764 (2012).

8. Lau, W. Y., Lai, E. C. \& Yu, S. C. in Hepatocellular Carcinoma (ed. W. Y. Lau) 739-760 (World Scientific Publishing, 2008)

9. Huang, J. et al. Exome sequencing of hepatitis $B$ virus-associated hepatocellular carcinoma. Nat. Genet. 44, 1117-1121 (2012).

10. Tao, Y. et al. Rapid growth of a hepatocellular carcinoma and the driving mutations revealed by cell-population genetic analysis of wholegenome data. Proc. Natl Acad. Sci. USA 108, 12042-12047 (2011).

11. Nomoto, S. et al. Hypermethylation of multiple genes as clonal markers in multicentric hepatocellular carcinoma. Br. J. Cancer 97 , 1260-1265 (2007). 